

\title{
ANALYSIS OF ESTIMATORS FOR ADAPTIVE KINETIC MONTE CARLO
}

\author{
David ARistoff, SAmuel T. Chill And Gideon Simpson
}

\begin{abstract}
Adaptive Kinetic Monte Carlo combines the simplicity of Kinetic Monte Carlo (KMC) with a saddle point search algorithm based on Molecular Dynamics (MD) in order to simulate metastable systems. Key to making Adaptive KMC effective is a stopping criterion for the saddle point search. In this work, we examine a criterion of S. T. Chill and G. Henkelman (J. Chem. Phys. 140 (2014), no. 21, 214110), which is based on the fraction of total reaction rate found instead of the fraction of observed saddles. The criterion uses the Eyring-Kramers law to estimate the reaction rate at the MD search temperature. We also consider a related criterion that remains valid when the Eyring-Kramers law is not. We examine the mathematical properties of both estimators and prove their mean square errors are well behaved, vanishing as the simulation continues to run.
\end{abstract}

\section{Introduction}

An outstanding problem in theoretical materials science and chemistry is how to reach laboratory time scales of microseconds $\left(10^{-6} \mathrm{~s}\right)$ and longer using models, based on Molecular Dynamics (MD), which resolve the atomistic time scale of femtoseconds $\left(10^{-15} \mathrm{~s}\right)$. Much of this scale separation is due to the presence of metastable regions in the configuration space of the system. In such regions, often defined by local minima of an energy landscape, the system stays close to a particular configuration, such as a local minima, before crossing into some other metastable region associated with a different configuration. Consequently, during much of a direct MD simulation, the system is close to one metastable region or another. It exhibits dynamics akin to a continuous time random walk on the set of metastable states, with comparatively long waiting times.

Since much of the physical significance of these systems is characterized by the sequence of visited metastable states and the time spent in each, there have been a variety of efforts to systematically coarse grain the MD trajectory into a more

Aristoff was supported by the National Science Foundation via the award NSF-DMS-1522398. Simpson was supported by US Department of Energy Award DE-SC0012733. He also thanks P. Hitczenko for helpful discussions.

MSC2010: 65C05, 65C20, 82C80.

Keywords: kinetic Monte Carlo, molecular dynamics, stopping time. 
computationally efficient continuous time random walk. A. F. Voter has proposed three methods, Parallel Replica Dynamics, Hyperdynamics, and Temperature Accelerated Dynamics, which can overcome metastability through intelligent usage of the primitive Langevin dynamics $[14 ; 16]$. In recent years, significant effort has been made to understand and quantify the approximations in these methods and extend their applicability $[2 ; 3 ; 1 ; 5 ; 11 ; 12 ; 15]$.

Another approach to the problem is Kinetic Monte Carlo (KMC), and this will be the focus of this work. Let us assume our system is governed by a potential energy $V(x), x \in \mathbb{R}^{d}$ at inverse temperature $\beta$. Furthermore, we assume that we have partitioned configuration space into an at most countable set of metastable states, $\Omega_{i}$, associated with local minima $m_{i}$ of $V$. The system can go from metastable state $i$ to metastable state $j$ if there is a saddle point, $s_{i j}$, of $V(x)$ joining $\Omega_{i}$ and $\Omega_{j}$. For conciseness, we will assume there is a single saddle point joining two given adjacent metastable states, though, in general, there may be multiple pathways.

In traditional KMC, before a simulation is run, one must identify the metastable states, their connectivity (i.e., which ones are joined by saddle points), and the reaction rates of each such connection. Given all of this information, KMC is very cheap to simulate. A single random number is generated and used to select one of the possible reactions, the system migrates into the new metastable region, and the algorithm repeats.

Unfortunately, such complete details of the metastable states and their connectivity are, a priori, unavailable in all but the simplest low-dimensional systems. This has motivated the development of Adaptive Kinetic Monte Carlo (AKMC) $[6 ; 17 ; 18]$. In AKMC, the system starts in some metastable region $\Omega_{i}$. Saddle points associated with $\Omega_{i}$ are then sought via a saddle point search algorithm that successively finds $s_{i j}$. Reaction rates for each such saddle can be estimated by the Eyring-Kramers law [8]:

$$
k_{i j}=g_{i j} \exp \left[-\beta\left(V\left(s_{i j}\right)-V\left(m_{i}\right)\right)\right],
$$

where, writing $\lambda_{1}$ for the sole negative eigenvalue of $\nabla^{2} V\left(s_{i j}\right)$,

$$
g_{i j}=\frac{\left|\lambda_{1}\right|}{\pi} \sqrt{\left|\frac{\operatorname{det} \nabla^{2} V\left(m_{i}\right)}{\operatorname{det} \nabla^{2} V\left(s_{i j}\right)}\right|} .
$$

Once a sufficient number of saddles associated with $\Omega_{i}$ have been identified, the problem is treated by using traditional KMC with the thus far identified reactions and their rates; this process then repeats in the next metastable region. Two things are needed to proceed with AKMC:

(1) a saddle point search algorithm;

(2) a stopping criterion. 
In this work, we will consider the question of the stopping criterion, provided our saddle point search algorithm satisfies certain assumptions. Our analysis will focus on estimators similar to the one introduced by Chill and Henkelman [6]. We call these Chill-type estimators.

In [6], the authors searched for saddle points out of each metastable state using high-temperature MD. For concreteness, consider the Brownian dynamics in $\mathbb{R}^{d}$ :

$$
d X_{t}=-\nabla V\left(X_{t}\right) d t+\sqrt{2 \beta^{-1}} d W_{t} .
$$

The aim is to model the dynamics at low temperature $\beta=\beta^{\text {lo }}$. Starting at $X_{0} \in \Omega_{i}$, integrate (1-2) at a higher temperature $\beta=\beta^{\text {hi }}$ (i.e., $\beta^{\text {lo }}>\beta^{\text {hi }}$ ) until the trajectory leaves $\Omega_{i}$. Using the higher temperature $\beta^{\text {hi }}$ allows an escape to occur more quickly. After the trajectory leaves $\Omega_{i}$, one of the saddle points $s_{i j}$ is identified with this pathway using, for instance, the nudged elastic band method [10;9], and the low-temperature reaction rate is computed using (1-1) with $\beta=\beta^{\text {lo }}$. This is then repeated, with a new initial condition chosen in $\Omega_{i}$. Throughout, the cumulative simulation time is recorded.

Other saddle point search algorithms have been proposed, including the dimer method and the string method [13; 7]. In our analysis, the key property that we need to hold true for all of our search methods is the following. Let

$$
N_{i j}(t)=\text { Number of times saddle } s_{i j} \text { has been found by time } t \text {. }
$$

Then for fixed $i$, during a saddle point search, the $N_{i j}(t)$ are independent, with respect to $j$, Poisson processes. We prove below that this holds for a carefully performed saddle point search via integration of (1-2).

This article is organized as follows. We describe the saddle point search in detail in Section 2, and prove some of its properties, including the above condition on $N_{i j}(t)$, in Section 3. In Section 4 we introduce stopping criteria for the saddle point search, and in Section 5 we analyze these criteria. Section 6 contains proofs of some of the estimates in Section 5. In Section 7 we make some concluding remarks.

\section{Notation and saddle point search algorithm}

Here and throughout $\left(X_{t}\right)$ is Brownian dynamics, that is, a stochastic process satisfying (1-2). For simplicity we fix a single metastable set $\Omega \equiv \Omega_{i}$ and suppress the index $i$ in all of our notation from the introduction. For our purposes, $V$ is smooth, and $\Omega$ is an (open) basin of attraction of $V$ with respect to the gradient dynamics $d y / d t=-\nabla V(y)$. We assume that $\partial \Omega$ is partitioned into finitely many disjoint (measurable) subsets, called pathways and labeled $1,2, \ldots, N$, such that each pathway $j$ contains a unique saddle point $s_{j}$ of $V$. When $\left(X_{t}\right)$ leaves $\Omega$, it must exit through one of the pathways $1,2, \ldots, N$. 
The algorithm, as well as our analysis, depends heavily on the quasistationary distribution (QSD) for $\left(X_{t}\right)$ in $\Omega$, which we denote by $v$. The QSD $v$ is a probability measure that is locally invariant for $\left(X_{t}\right)$, in the sense that it is invariant conditionally on the event that $\left(X_{t}\right)$ remains in $\Omega$ :

Definition 2.1. The QSD for $\left(X_{t}\right)$ in $\Omega$ is a probability measure $v$ supported in $\Omega$ such that for all $t>0$,

$$
v(\cdot)=\mathbb{P}\left(X_{t} \in \cdot \mid X_{0} \sim v, X_{s} \in \Omega \text { for all } s \in[0, t]\right) .
$$

Of course $v$ depends on $\Omega$, but for simplicity we do not indicate this explicitly. It has been shown [11] that $v$ exists, is unique, and satisfies

$$
v(A)=\lim _{n \rightarrow \infty} \mathbb{P}\left(X_{t} \in A \mid X_{s} \in A \text { for } s \in[0, t]\right), \quad \text { for all } A \subset \Omega .
$$

Moreover this convergence is exponentially fast, uniformly in $A$. Equation (2-1) leads to simple algorithms for sampling $v$, based on the idea that a sample can be obtained from the endpoint of a trajectory of $\left(X_{t}\right)$ that has remained in $\Omega$ for a sufficiently long time; see [5] for details.

We are now ready to state the high-temperature saddle point search algorithm. Versions of this algorithm have been used previously; see for instance [6] and references therein. The search runs at a user-specified "high" (inverse) temperature $\beta^{\text {hi }}$. Below we write $\nu$ for the QSD in $\Omega$ at temperature $\beta=\beta^{\text {hi }}$. We also write

$$
H(t)= \begin{cases}0, & t<0 \\ 1, & t \geq 0\end{cases}
$$

for the Heaviside unit step function.

Algorithm 2.2. Set $N_{j}(t) \equiv 0$ for $t \geq 0$ and $j=1, \ldots, N$. Let $M$ be the current cycle of the algorithm, and $t_{\text {sim }}$ the simulation clock. Initialize $M=1$ and $t_{\text {sim }}=0$, and iterate the following:

1. Generate a sample $x_{M}$ from $v$. During this step $t_{\text {sim }}$ is stopped.

2. Starting at $X_{0}=x_{M}$, evolve $\left(X_{t}\right)$ at $\beta=\beta^{\text {hi }}$ until it first leaves $\Omega$, say at time $t=\tau^{(M)}$ through pathway $I^{(M)}$. The simulation clock $t_{\text {sim }}$ is running during this step, and the stopping criterion is continuously checked. If at some time $t_{\text {sim }}$ the criterion is met, the algorithm stops.

3. If $I^{(M)}=j$, update $N_{j}(t)=N_{j}(t)+H\left(t-t_{\text {sim }}\right)$ for $t \geq 0$ and record the saddle point $s_{j}$. Then update $M=M+1$. During this step $t_{\text {sim }}$ is stopped.

It is not necessary to know $N$, and the pathways can be given labels according to the order in which they are found. The simulation clock is cumulative, and it only increases in Step 2. In particular, during the $M$-th cycle of the algorithm, $t_{\text {sim }}$ increases by $\tau^{(M)}$. The stopping criterion will be described in Section 4. Below 
we write $t_{\text {sim }}$ for the final value of the simulation clock in the algorithm, that is, its value when the simulation is stopped. To refer to a generic simulation clock time we write $t$. Thus, $0 \leq t \leq t_{\text {sim }}$ and when the algorithm stops, $N_{j}(t)$ is the number of times an exit through pathway $j$ has been observed by time $t$. Below we write $N_{j}(t)$ for its final value when the algorithm stops. We will also use the following notation:

$$
\chi_{j}(t)=\mathbb{1}_{N_{j}(t) \geq 1}, \quad N(t)=\sum_{j=1}^{N} N_{j}(t) .
$$

That is, $\chi_{j}(t)=1$ if an exit through pathway $j$ has been observed at least once by time $t$, and is 0 otherwise; $N(t)$ is the total number of exits observed by time $t$.

\section{Properties of the saddle point search}

Our first result follows immediately from properties of the QSD established in [11]. Theorem 3.1. Suppose that in step 1 in the M-th cycle of Algorithm 2.2, $x_{M}$ is a random variable with distribution $v$. Then:

(i) $\tau^{(M)}$ is exponentially distributed with mean $\kappa^{-1}: \mathbb{P}\left(\tau^{(j)}>t\right)=\exp (-\kappa t)$.

(ii) $\tau^{(M)}$ and $I^{(M)}$ are independent.

Theorem 3.1 then leads to the following.

Theorem 3.2. Suppose that in step 1 of Algorithm 2.2, $x_{1}, x_{2}, \ldots$ are iid with common distribution $v$. Then:

(i) $\{N(t)\}_{0 \leq t \leq t_{\text {sim }}}$ is a Poisson process with parameter $\kappa$.

(ii) $\left\{N_{j}(t)\right\}_{0 \leq t \leq t_{\text {sim }}}^{j=1, \ldots, N}$ are independent Poisson processes with parameters

$$
\kappa_{j}:=\kappa p_{j}, \quad p_{j}:=\mathbb{P}\left(I^{(1)}=j\right) .
$$

Proof. Let $(\tilde{N}(s))_{s \geq 0}$ be a Poisson process with parameter $\kappa$, which we denote by $\tilde{N}(s)$ for brevity. Label each arrival time of $\tilde{N}(s)$ with a pathway $j$ according to the distribution $p_{j}$, independently of the other arrival times, and let $\tilde{N}_{j}(s)$ be the process with arrivals labeled by $j$. Then for $r, s \geq 0$ and $m_{1}, \ldots, m_{N} \geq 0$,

$$
\begin{aligned}
\mathbb{P}\left(\bigcap_{j=1}^{N}\left\{\tilde{N}_{j}(r+s)-\tilde{N}_{j}(r)=m_{j}\right\}\right) \\
\quad=\mathbb{P}\left(N(r+s)-N(r)=\sum_{j=1}^{N} m_{j}\right)\left(\begin{array}{c}
m_{1}+\cdots+m_{N} \\
m_{1}, \ldots, m_{N}
\end{array}\right) \prod_{j=1}^{N} p_{j}^{m_{j}} \\
=\prod_{j=1}^{N} \frac{e^{-\kappa p_{j} s}\left(\kappa p_{j} s\right)^{m_{j}}}{m_{j} !} .
\end{aligned}
$$


By summing over all $m_{i} \geq 0$ for $i \neq j$ in the last expression above, we see that for fixed $r, s \geq 0$, the increment $\tilde{N}_{j}(r+s)-\tilde{N}_{j}(r)$ is Poisson distributed with mean $\kappa p_{j} s . \tilde{N}_{j}(s)$ also inherits independent increments from $\tilde{N}(s)$. This shows that $\tilde{N}_{j}(s)$ is a Poisson process with parameter $\kappa_{j}=\kappa p_{j}$. Moreover, (3-2) shows that $\tilde{N}_{j}(s), j=1, \ldots, N$, are independent.

Let us now relate $(\tilde{N}(s))_{s \geq 0}$ with $(N(s))_{0 \leq s \leq t_{\text {sim }}}$. For fixed $s \in\left[0, t_{\text {sim }}\right]$, the time marginal $N(s)$ is the largest $m$ such that $\tau^{(1)}+\cdots+\tau^{(m)} \leq s$. Together with part (i) of Theorem 3.1, this shows that on $\left[0, t_{\text {sim }}\right],(N(s))_{0 \leq s \leq t_{\text {sim }}}$ and $(\tilde{N}(s))_{s \geq 0}$ are Poisson processes with the same law. By part (ii) of Theorem 3.1, it follows that the multivariate processes $\left(N_{j}(s)\right)_{0 \leq s \leq t_{s i m}}^{j=1, \ldots, N}$ and $\left(\tilde{N}_{j}(s)\right)_{0 \leq s \leq t_{\text {sim }}}^{j=1, \ldots, N}$ have the same law. This establishes the result.

\section{Chill-type estimators and stopping criteria}

The purpose of the high-temperature saddle point search (Algorithm 2.2) is to locate "enough" of the low-temperature rate corresponding to the metastable set $\Omega$. More precisely, at a low temperature corresponding to $\beta=\beta^{\text {lo }}$, the first exit time of $X_{t}$ from $\Omega$ is approximately exponentially distributed with mean $\left(k_{1}+\cdots+k_{N}\right)^{-1}$, where $k_{j}=k_{j}^{\text {lo }}$ is given by the Eyring-Kramers law (1-1) at $\beta=\beta^{\text {lo }}$ (recall the subscript $i$ has been suppressed). See [4] and references therein for rigorous results in this direction. The $k_{j}$ are then exponential rates associated with leaving $\Omega$ through pathway $j$ at low temperature $\beta^{\text {lo }}$. The proportion of low-temperature rate found by time $t$ in Algorithm 2.2 is

$$
R(t):=\frac{\sum_{j=1}^{N} \chi_{j}(t) k_{j}}{\sum_{j=1}^{N} k_{j}} .
$$

The expected value of $R(t)$ is

$$
\mathbb{E}[R(t)]=\bar{R}(t):=\frac{\sum_{j=1}^{N} p_{j}(t) k_{j}}{\sum_{j=1}^{N} k_{j}},
$$

where

$$
p_{j}(t):=\mathbb{E}\left[\chi_{j}(t)\right]=1-\exp \left(-\kappa_{j} t\right) .
$$

Here $\kappa_{j}$ is defined as in Theorem 3.2 at temperature $\beta=\beta^{\text {hi }}$. The idea behind Chilltype estimators is that when $R(t)$ is sufficiently close to 1 , the high-temperature saddle point search can stop. There are two obstacles to this idea.

The first is that, at any time during Algorithm 2.2, it is unlikely that all saddle points have been found. This problem is remedied by replacing $k_{j}$ in (4-1) with $\chi_{j}(t) k_{j}$, which is computable once pathway $j$ has been found during the simulation. The second obstacle is that an exact formula for $p_{j}(t):=\mathbb{E}\left[\chi_{j}(t)\right]$ will not be known 
in practice. Chill-type estimators overcome the latter obstacle by using one of the following approximations:

$$
\begin{array}{ll}
\tilde{p}_{j}(t):=1-\exp \left[-k_{j}^{\mathrm{hi}} t\right], & k_{j}^{\mathrm{hi}} \text { given by Eyring-Kramers }(1-1) \text { at } \beta=\beta^{\mathrm{hi}}, \\
\hat{p}_{j}(t):=1-\exp \left[-\hat{N}_{j}(t)\right], & \hat{N}_{j}(t):= \begin{cases}N_{j}(t), & N_{j}(t) \geq 2, \\
0, & \text { else. }\end{cases}
\end{array}
$$

We have used the superscript hi to emphasize that the rate in (4-4) is computed at temperature $\beta^{\text {hi }}$ (whereas $k_{j}$ is computed at low temperature $\beta^{\text {lo }}$ ). Also note that $\tilde{p}_{j}(t)$ is a physical estimate of $\mathbb{E}\left[\chi_{j}(t)\right]$ based on Eyring-Kramers, while $\hat{p}_{j}(t)$ is a (biased) Monte Carlo estimator. From (4-4) we obtain the following estimators for $R(t)$ :

$$
\tilde{R}(t):=\frac{\sum_{j=1}^{N} \tilde{p}_{j}(t) \chi_{j}(t) k_{j}}{\sum_{j=1}^{N} \chi_{j}(t) k_{j}}, \quad \hat{R}(t):=\frac{\sum_{j=1}^{N} \hat{p}_{j}(t) \chi_{j}(t) k_{j}}{\sum_{j=1}^{N} \chi_{j}(t) k_{j}} .
$$

$R(t), \tilde{R}(t)$, and $\hat{R}(t)$ are all random, while $\bar{R}(t)$ is deterministic. Both $\tilde{R}(t)$ and $\hat{R}(t)$ are explicitly computable at time $t$ during the saddle point search. See [6] for further discussion of $\tilde{R}(t)$. To our knowledge $\hat{R}(t)$ has not appeared before in the literature. We emphasize that $\hat{R}(t)$ may be used at any temperature $\beta^{\text {hi }}$, while $\tilde{R}(t)$ is limited by the fact that it gives reasonable estimates of $R(t)$ only at (relatively low) temperatures where the Eyring-Kramers law holds.

After choosing $\tilde{R}(t)$ or $\hat{R}(t)$ as the preferred estimator, the stopping criterion can now be defined as follows: for a user-specified parameter $\epsilon>0$, stop Algorithm 2.2 in Step 3 if and only if

$$
\tilde{R}(t)>1-\epsilon \text { or } \hat{R}(t)>1-\epsilon,
$$

respectively. In Section 5 we give rigorous estimates of the bias and variance of the estimators $\tilde{R}(t)$ and $\hat{R}(t)$. These estimates show that, as $t$ increases, when the algorithm stops, on average at least $(1-\epsilon) \%$ of the low-temperature rate has been found.

\section{Analysis}

The approximation $\tilde{p}_{j}(t)$ of $p_{j}(t)$ is usually considered valid when

$$
\beta^{\text {hi }} \ll V\left(s_{j}\right)-V(m),
$$

with $m$ the minimizer of $V$ in $\Omega$. To the authors' knowledge, rigorous results are scarce except when

$$
s_{j}=\operatorname{argmin}_{s_{1}, \ldots, s_{N}} V\left(s_{j}\right)-V(m) ;
$$

see [4] and references therein. However, the following is a consequence of results in [2]: 
Theorem 5.1. Suppose $\Omega=(a, b)$ is an interval and $V$ is a Morse potential. Then for each $t>0$,

$$
\frac{1-\tilde{p}_{j}(t)}{1-p_{j}(t)}=1+O\left(1 / \beta^{\mathrm{hi}}\right) \quad \text { as } \beta^{\mathrm{hi}} \rightarrow \infty, \quad j=1,2 .
$$

Proof. An examination of the proof of Theorem 4.1 of [2] shows that for $j=1,2$,

$$
k_{j}^{\mathrm{hi}} / \kappa_{j}=1+O\left(1 / \beta^{\mathrm{hi}}\right) \quad \text { as } \beta^{\mathrm{hi}} \rightarrow \infty,
$$

where $k_{j}^{\text {hi }}$ is as in (4-4), and $\kappa_{j}$ is as in Theorem 3.2 at temperature $\beta=\beta^{\text {hi }}$. The result follows.

We next examine the approximation $\hat{p}(t)$ of $p(t)$.

Theorem 5.2. Conditionally on $N(t) \geq 1, \hat{N}_{j}(t)$ is an unbiased estimator for $\kappa_{j} t$ :

$$
\mathbb{E}\left[\hat{N}_{j}(t) \mid N(t) \geq 1\right]=\kappa_{j} t .
$$

Also conditionally on $N(t) \geq 1, \hat{p}_{j}(t)$ is a conservative estimate of $p_{j}(t)$ :

$$
\mathbb{E}\left[\hat{p}_{j}(t) \mid N_{j}(t) \geq 1\right] \leq p_{j}(t) .
$$

Proof. Recall that $N_{j}(t)$ is a Poisson process with parameter $\kappa_{j}$. Thus,

$$
\begin{aligned}
\mathbb{E}\left[\hat{N}_{j}(t) \mid N_{j}(t) \geq 1\right] & =\left(1-e^{-\kappa_{j} t}\right)^{-1} \sum_{n=2}^{\infty} n \frac{\left(\kappa_{j} t\right)^{n} e^{-\kappa_{j} t}}{n !} \\
& =\frac{\kappa_{j} t}{1-e^{-\kappa_{j} t}} \sum_{n=1}^{\infty} \frac{\left(\kappa_{j} t\right)^{n} e^{-\kappa_{j} t}}{n !}=\kappa_{j} t .
\end{aligned}
$$

Since $x \mapsto 1-e^{-x}$ is a concave function, the second statement of the theorem follows from Jensen's inequality.

The reason that we consider conditional expectations in Theorem 5.2 is that Algorithm 2.2 cannot stop before $N(t) \geq 1$. Thus, we want estimates conditioned on that event. We call $\hat{p}_{j}(t)$ a conservative estimate for $p_{j}(t)$ because it is a lower bound on average, so that using $\hat{p}_{j}(t)$ in place of $p_{j}(t)$ leads to a larger average stopping time for Algorithm 2.2.

Before proceeding we define, for real-valued random variables $X$ and $Y$,

$$
\operatorname{Bias}(X, Y):=\mathbb{E}[X-Y], \quad \operatorname{MSE}(X, Y):=\operatorname{Bias}(X, Y)^{2}+\operatorname{Var}(X) .
$$

Observe that the mean square error is not symmetric in its arguments. 
Theorem 5.3. Write $q_{j}(t)=1-p_{j}(t)=\exp \left[-\kappa_{j} t\right]$ and $K=k_{1}+\cdots+k_{N}$. For the estimator $\tilde{R}(t)$,

$|\operatorname{Bias}(\tilde{R}(t), R(t))| \leq N \max _{j}\left|\operatorname{Bias}\left(\tilde{p}_{j}(t), p_{j}(t)\right)\right|+\frac{K}{\min _{j} k_{j}} \bar{R}(t) \max _{j} q_{j}(t)$,

$$
\operatorname{Var}(\tilde{R}(t)) \leq 4 \frac{K^{2}}{\min _{j} k_{j}^{2}} \bar{R}(t)^{2} \max _{j} q_{j}(t)
$$

$\operatorname{MSE}(\tilde{R}(t), R(t)) \leq 2 N^{2} \max _{j} \operatorname{MSE}\left(\tilde{p}_{j}(t), p_{j}(t)\right)$

$$
+\frac{K^{2}}{\min _{j} k_{j}^{2}}\left(2 \max _{j} q_{j}(t)+4\right) \bar{R}(t)^{2} \max _{j} q_{j}(t) .
$$

For the estimator $\hat{R}(t)$,

$|\operatorname{Bias}(\hat{R}(t), R(t))| \leq N \max _{j}\left|\operatorname{Bias}\left(\hat{p}_{j}(t), p_{j}(t)\right)\right|+\frac{K}{\min _{j} k_{j}} \bar{R}(t) \max _{j} q_{j}(t)$,

$$
\begin{aligned}
\operatorname{Var}(\hat{R}(t)) \leq \frac{2 K^{2}}{\min _{j} k_{j}^{2}} \bar{R}(t)^{2} \max _{j} & q_{j}(t) \\
& +\left(1+2 N^{2} \max _{j} q_{j}(t)\right) \max _{j} \operatorname{Var}\left(\hat{p}_{j}(t)\right),
\end{aligned}
$$

$\operatorname{MSE}(\hat{R}(t), R(t)) \leq\left(1+N^{2}+2 N^{2} \max _{j} q_{j}(t)\right) \max _{j} \operatorname{MSE}\left(\hat{p}_{j}(t), p_{j}(t)\right)$

$$
+\frac{4 K^{2}}{\min _{j} k_{j}^{2}} \bar{R}(t)^{2}\left(1+\max _{j} q_{j}(t)\right) \max _{j} q_{j}(t) .
$$

Here, all maxima and minima are taken over $j \in\{1, \ldots, N\}$.

Proof. We give proofs in Section 6 below.

We note that some of the bounds in Theorem 5.3 have been loosened so that simpler expressions are obtained. This will become clear in the derivation of the bounds in Section 6 below. We highlight that the bias is bounded by the bias of the estimate of $p_{j}(t)$, together with another term representing an "inherent" bias associated with $\bar{R}(t)$. This second term may be approximated by noting that $|\bar{R}(t)|<1$ for all $t$ and, due to Theorem 5.1, we expect $q_{j}(t)$ can be estimated by the known function $\tilde{p}_{j}(t)$ or $\hat{p}_{j}(t)$.

\section{Estimates}

In this section we give a proof of Theorem 5.3. Recall that $q_{j}(t):=1-p_{j}(t)$ and $K:=\sum_{j=1}^{N} k_{j}$ is the total reaction rate. For brevity, we will sometimes suppress the $t$ dependence in our expressions. Also, all sums are over $1, \ldots, N$ unless otherwise indicated. 


\section{A. Preliminary calculations. Observe that}

$\operatorname{Bias}(\tilde{R}(t), R(t))=\operatorname{Bias}(\tilde{R}(t), \bar{R}(t)), \quad \operatorname{MSE}(\tilde{R}(t), R(t))=\operatorname{MSE}(\tilde{R}(t), \bar{R}(t))$, and similarly for $\hat{R}(t)$; this fact will be used below without comment. There are a few expressions that will show up repeatedly in the analyses of both $\tilde{R}$ and $\hat{R}$. We analyze them here for simplicity. Let

$$
\xi_{i}=k_{i}+\sum_{m \neq i} k_{m} \chi_{m}
$$

We make the following calculations:

$$
\begin{gathered}
k_{i} \leq \xi_{i} \leq K, \\
\mathbb{E}\left[\xi_{i}\right]=k_{i}+\sum_{m \neq i} p_{m} k_{m}=K-\sum_{m \neq i} q_{m} k_{m} .
\end{gathered}
$$

A lower bound on this can be obtained from Jensen's inequality,

$$
\mathbb{E}\left[\xi_{i}^{-1}\right] \geq \mathbb{E}\left[\xi_{i}\right]^{-1}=\frac{1}{K-\sum_{m \neq i} q_{m} k_{m}} \geq \frac{1}{K}+\frac{1}{K^{2}} \sum_{m \neq i} k_{m} q_{m},
$$

while an upper bound can be obtained from the Edmundson-Madansky inequality,

$$
\mathbb{E}\left[\xi_{i}^{-1}\right] \leq \frac{1}{k_{i}} \frac{K-\mathbb{E}\left[\xi_{i}\right]}{K-k_{i}}+\frac{1}{K} \frac{\mathbb{E}\left[\xi_{i}\right]-k_{i}}{K-k_{i}}=\frac{1}{K}+\frac{1}{k_{i} K} \sum_{m \neq i} k_{m} q_{m} .
$$

In the same way,

$$
\mathbb{E}\left[\xi_{i}^{-2}\right] \geq \mathbb{E}\left[\xi_{i}\right]^{-2}=\frac{1}{\left(K-\sum_{m \neq i} q_{m} k_{m}\right)^{2}} \geq \frac{1}{K^{2}}+\frac{2}{K^{3}} \sum_{m \neq i} q_{m} k_{m},
$$

and

$$
\mathbb{E}\left[\xi_{i}^{-2}\right] \leq \frac{1}{k_{i}^{2}} \frac{K-\mathbb{E}\left[\xi_{i}\right]}{K-k_{i}}+\frac{1}{K^{2}} \frac{\mathbb{E}\left[\xi_{i}\right]-k_{i}}{K-k_{i}}=\frac{1}{K^{2}}+\frac{K+k_{i}}{k_{i}^{2} K^{2}} \sum_{m \neq i} q_{m} k_{m} .
$$

Therefore,

$$
\operatorname{Var}\left(\xi_{i}^{-1}\right) \leq\left(\frac{K+k_{i}}{k_{i}^{2} K^{2}}-\frac{2}{K^{3}}\right) \sum_{m \neq i} q_{m} k_{m} \leq \frac{2}{K k_{i}^{2}} \sum_{m \neq i} q_{m}(t) k_{m},
$$

where we have lost some of the estimate in the last inequality for the sake of conciseness.

6B. Estimates for $\tilde{\boldsymbol{R}}$. Below it is useful to notice that

$$
\tilde{R}(t)=\sum_{i=1}^{N} \frac{\tilde{p}_{i}(t) \chi_{i}(t) k_{i}}{k_{i}+\sum_{m \neq i} \chi_{m}(t) k_{m}}=\sum_{i} \frac{\tilde{p}_{i} \chi_{i} k_{i}}{\xi_{i}} .
$$


6B1. Bias. We begin with the direct calculation

$$
\begin{aligned}
\mathbb{E}[\tilde{R}-\bar{R}] & =\sum_{i=1}^{N} \mathbb{E}\left[\frac{\chi_{i} \tilde{p}_{i} k_{i}}{\xi_{i}}-\frac{\chi_{i} k_{i}}{K}\right] \\
& =\sum_{i=1}^{N}\left(\tilde{p}_{i}-p_{i}\right) \mathbb{E}\left[\frac{\chi_{i} k_{i}}{\xi_{i}}\right]+\sum_{i=1}^{N} \mathbb{E}\left[\frac{\chi_{i} p_{i} k_{i}}{\xi_{i}}-\frac{\chi_{i} k_{i}}{K}\right] \\
& =\sum_{i=1}^{N}\left(\tilde{p}_{i}-p_{i}\right) \mathbb{E}\left[\frac{\chi_{i} k_{i}}{\xi_{i}}\right]+\sum_{i=1}^{N} \underbrace{\mathbb{E}\left[\frac{K p_{i}}{\xi_{i}}-1\right]}_{\equiv b_{i}} \frac{p_{i} k_{i}}{K} .
\end{aligned}
$$

Using (6-3) and (6-4),

$$
\frac{1}{K} \sum_{m \neq i} k_{m} q_{m}-q_{i} \leq b_{i} \leq \frac{1}{k_{i}} \sum_{m \neq i} k_{m} q_{m}-q_{i}
$$

Thus,

$$
\left|\sum_{i=1}^{N} b_{i} \frac{p_{i} k_{i}}{K}\right| \leq \sum_{i=1}^{N}\left(\sum_{j=1}^{N} \frac{k_{j}}{k_{i}} q_{j}\right) \frac{p_{i}(t) k_{i}}{K} \leq \frac{K \max _{j} q_{j}(t)}{\min _{j} k_{j}} \bar{R}(t) .
$$

Combining the above expressions gives

$$
|\operatorname{Bias}(\tilde{R}(t), \bar{R}(t))| \leq N \max _{i}\left|\tilde{p}_{i}(t)-p_{i}(t)\right|+\frac{K \max _{i} q_{i}(t)}{\min _{i} k_{i}} \bar{R}(t) .
$$

6B2. Variance. For the variance, we first write

$$
\tilde{R}-\mathbb{E}[\tilde{R}]=\sum_{i=1}^{N}\left(\frac{\chi_{i}}{\xi_{i}}-\mathbb{E}\left[\frac{\chi_{i}}{\xi_{i}}\right]\right) \tilde{p}_{i} k_{i}
$$

Hence,

$$
\operatorname{Var}(\tilde{R}(t))=\sum_{i, j=1}^{N} k_{i} k_{j} \tilde{p}_{i} \tilde{p}_{j} \underbrace{\operatorname{Cov}\left(\frac{\chi_{i}}{\xi_{i}}, \frac{\chi_{j}}{\xi_{j}}\right)}_{\equiv v_{i j}} .
$$

Since $v_{i j} \leq \sqrt{v_{i i}} \sqrt{v_{j j}}$, it will be sufficient for us to analyze the diagonal terms. By Theorem 3.2, $\chi_{i}$ and $\xi_{i}$ are independent. Thus

$$
v_{i i}=\mathbb{E}\left[\chi_{i}\right]^{2} \operatorname{Var}\left(\xi_{i}^{-1}\right)+\mathbb{E}\left[\xi_{i}^{-1}\right]^{2} \operatorname{Var}\left(\chi_{i}\right)+\operatorname{Var}\left(\xi_{i}^{-1}\right) \operatorname{Var}\left(\chi_{i}\right) .
$$


Using (6-6) and (6-7),

$$
\begin{aligned}
v_{i i} & \leq p_{i} \operatorname{Var}\left(\xi_{i}^{-1}\right)+p_{i} q_{i} \mathbb{E}\left[\xi_{i}^{-2}\right] \\
& \leq p_{i}\left(\frac{K+k_{i}}{k_{i}^{2} K^{2}}-\frac{2}{K^{3}}\right) \sum_{m \neq i} q_{m} k_{m}+p_{i} q_{i}\left(\frac{1}{K^{2}}+\frac{K+k_{i}}{k_{i}^{2} K^{2}} \sum_{m \neq i} q_{m} k_{m}\right) \\
& \leq \frac{p_{i} q_{i}}{K^{2}}+\frac{4 p_{i}}{k_{i}^{2} K} \sum_{m \neq i} q_{m} k_{m} \leq \frac{4 p_{i}}{k_{i}^{2}} \max _{j} q_{j} \leq \frac{4}{k_{i}^{2}} \max _{j} q_{j}(t) \\
& \leq \frac{4}{\min _{j} k_{j}^{2}} \max _{j} q_{j}(t) .
\end{aligned}
$$

We have made some sacrifices in the last inequalities in order to obtain a more concise expression. Consequently,

$$
\operatorname{Var}(\tilde{R}(t)) \leq \sum_{i, j=1}^{N} k_{i} k_{j} \tilde{p}_{i}(t) \tilde{p}_{j}(t) \sqrt{v_{i i}} \sqrt{v_{j j}} \leq \frac{4 K^{2}}{\min _{i} k_{i}^{2}} \bar{R}(t)^{2} \max _{i} q_{i}(t) .
$$

6B3. MSE. Combining (6-9) and (6-14), we then obtain $\operatorname{MSE}(\tilde{R}(t), \bar{R}(t)) \leq 2 N^{2} \max _{i}\left|\tilde{p}_{i}(t)-p_{i}(t)\right|^{2}$

$$
+\frac{K^{2}}{\min _{i} k_{i}^{2}}\left(2 \max _{i} q_{i}(t)+4\right) \bar{R}(t)^{2} \max _{i} q_{i}(t) .
$$

In this calculation, we see that the mean square error may ultimately be dominated by how well the $\tilde{p}_{i}$ approximate the $p_{i}$.

6C. Estimates for $\hat{\boldsymbol{R}}$. We begin by noting that, since $\hat{p}_{j}(t)=0$ if $\chi_{j}(t) \neq 1$,

$$
\hat{R}(t)=\sum_{j} \frac{\hat{p}_{j}(t) k_{j}}{k_{j}+\sum_{m \neq j} \chi_{m}(t) k_{j}} .
$$

6C1. Bias. We begin by writing

$$
\hat{R}-\bar{R}=\sum_{i=1}^{N}\left(\hat{p}_{i}-p_{i}\right) \frac{k_{i}}{\xi_{i}}+\sum_{i=1}^{N} \frac{k_{i} p_{i}}{\xi_{i}}-\frac{k_{i} p_{i}}{K},
$$

so that, after taking an expectation,

$$
\mathbb{E}[\hat{R}-\bar{R}]=\sum_{i=1} \mathbb{E}\left[\left(\hat{p}_{i}-p_{i}\right) \frac{k_{i}}{\xi_{i}}\right]+\sum_{i=1}^{N}\left(\mathbb{E}\left[\frac{K}{\xi_{i}}\right]-1\right) \frac{k_{i} p_{i}}{K} .
$$

Hence,

$$
|\operatorname{Bias}(\hat{R}(t), \bar{R}(t))| \leq N \max _{i}\left|\operatorname{Bias}\left(\hat{p}_{i}(t), p(t)\right)\right|+\frac{K}{\min _{i} k_{i}} \bar{R}(t) \max _{i} q_{i}(t),
$$


and we see that the observed bias is controlled by the biases of the approximate probabilities, $\hat{p}_{i}$, and the inherent bias of the Chill-type estimators.

6C2. Variance. For the variance, we have

$$
\operatorname{Var}(\hat{R})=\sum_{i, j=1}^{N} k_{i} k_{j} \underbrace{\operatorname{Cov}\left(\frac{\hat{p}_{i}}{\xi_{i}}, \frac{\hat{p}_{j}}{\xi_{j}}\right)}_{\equiv \hat{v}_{i j}} .
$$

As before, we only need to study the diagonal entries, and use Theorem 3.2 to obtain

$$
\begin{aligned}
\hat{v}_{i i} & =\mathbb{E}\left[\hat{p}_{i}\right]^{2} \operatorname{Var}\left(\xi_{i}^{-1}\right)+\mathbb{E}\left[\xi_{i}^{-1}\right]^{2} \operatorname{Var}\left(\hat{p}_{i}\right)+\operatorname{Var}\left(\hat{p}_{i}\right) \operatorname{Var}\left(\xi_{i}^{-1}\right) \\
& \leq \operatorname{Var}\left(\xi_{i}^{-1}\right)+\mathbb{E}\left[\xi_{i}^{-2}\right] \operatorname{Var}\left(\hat{p}_{i}\right) \\
& \leq \frac{2}{\min _{i} k_{i}^{2}} \max _{i} q_{i}+\left(\frac{1}{K^{2}}+\frac{2}{\min _{i} k_{i}^{2}} \max _{i} q_{i}\right) \operatorname{Var}\left(\hat{p}_{i}\right) \\
& \leq \frac{2}{\min _{i} k_{i}^{2}} \max _{i} q_{i}+\left(\frac{1}{K^{2}}+\frac{2}{\min _{i} k_{i}^{2}} \max _{i} q_{i}\right) \max _{i} \operatorname{Var}\left(\hat{p}_{i}\right) .
\end{aligned}
$$

We note that these estimates require full independence of $N_{j}(t)$ for $j=1, \ldots, N$, not just independence of the $\chi_{j}(t)$. Now,

$\operatorname{Var}(\hat{R}(t)) \leq \frac{2 K^{2}}{\min _{i} k_{i}^{2}} \bar{R}(t)^{2} \max _{i} q_{i}(t)+\left(1+2 N^{2} \max _{i} q_{i}(t)\right) \max _{i} \operatorname{Var}\left(\hat{p}_{i}(t)\right)$.

6C3. MSE. We can therefore express the mean square error of estimator $\hat{R}$ as

$$
\begin{aligned}
\operatorname{MSE}(\hat{R}(t), \bar{R}(t)) \leq & \frac{4 K^{2}}{\min _{i} k_{i}^{2}} \bar{R}(t)^{2}\left(1+\max _{i} q_{i}(t)\right) \max _{i} q_{i}(t) \\
& +\left(1+N^{2}+2 N^{2} \max _{i} q_{i}(t)\right) \max _{i} \operatorname{MSE}\left(\hat{p}_{i}(t), p_{i}(t)\right) .
\end{aligned}
$$

\section{Discussion}

We have considered three Chill-type estimators and shown them to be consistent. Their biases are small, relative to their variances, and thus we have good estimators of $R(t)$, the true fraction of the observed rate in the system. They represent a significant improvement over the original AKMC stopping criterion presented in [17]. Indeed, these prior approaches attempted to estimate the fraction of the saddles observed when, in fact, it is the fraction of the observed rate that is of fundamental importance.

As an example, we will compare the accuracy of both estimators using a test system that consists of saddle points $s_{j}$ corresponding to potential energy barriers 


$$
n=-\frac{1}{2}
$$

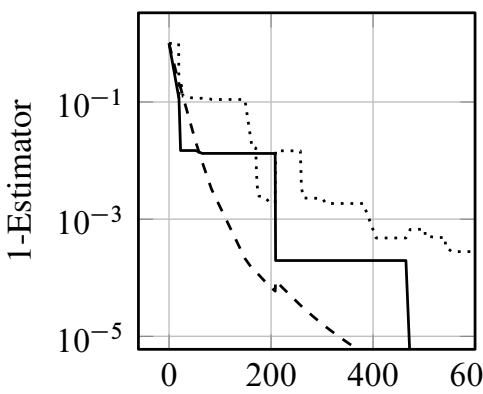

$n=0$

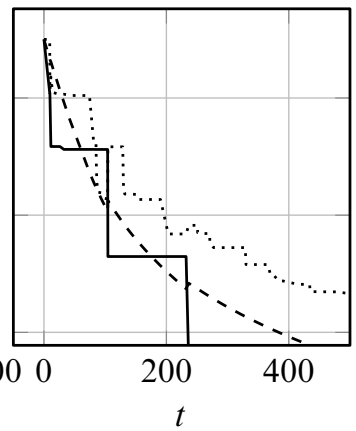

$$
n=\frac{1}{2}
$$

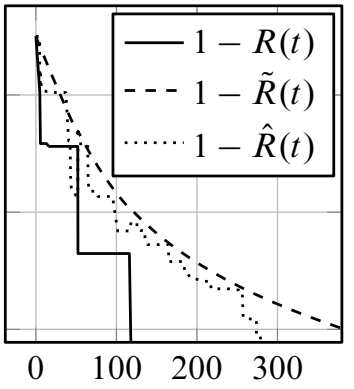

Figure 1. Comparison of the Chill-type estimators $\tilde{R}(t)$ and $\hat{R}(t)$ to the true expected proportion of the low-temperature rate found, $R(t)$, on a test system that can deviate from the Eyring-Kramer law.

$$
n=-\frac{1}{2}
$$

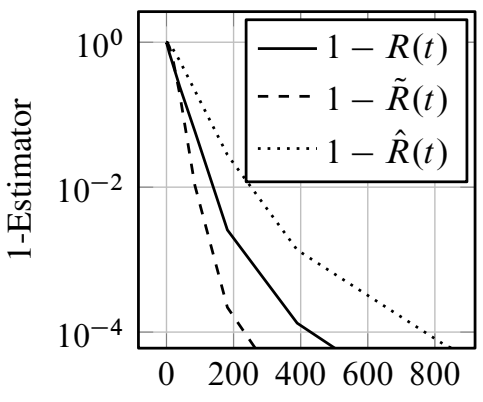

$n=0$

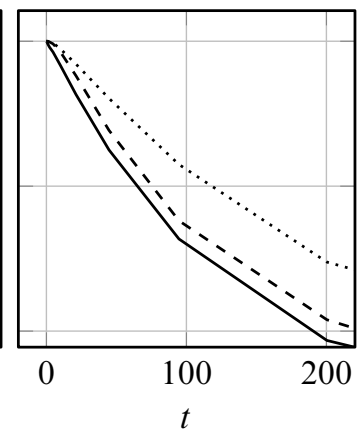

$n=\frac{1}{2}$

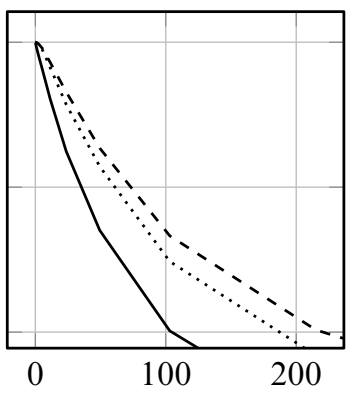

Figure 2. Comparison of the expected value of the Chill-type estimators $\tilde{R}(t)$ and $\hat{R}(t)$ to the true expected proportion of the low-temperature rate found, $R(t)$, on a test system that can deviate from the Eyring-Kramer law.

$V\left(s_{j}\right)-V(m)=1+\frac{4}{19} j$, for $j=0, \ldots, 19$. The test system has rates that obey a modified Arrhenius equation with the form

$$
\tilde{k}_{j}^{\mathrm{hi}}=\left(\frac{\beta^{\mathrm{lo}}}{\beta^{\mathrm{hi}}}\right)^{n} g_{j} \exp \left[\beta\left(V\left(s_{j}\right)-V(m)\right)\right] .
$$

Compare to Equation (1-1) (recall the subscript $i$ has been suppressed). The variable $n$ controls how the rates deviate from an unmodified Arrhenius rate law. When $n=0$ the modified rates $\tilde{k}_{j}^{\text {hi }}$ are equal to the unmodified rates $k_{j}^{\text {hi }}$, while when $\beta^{\text {hi }}<\beta^{\text {lo }}$, the modified rates are larger (resp. smaller) than the unmodified rates if $n>0$ (resp. $n<0)$.

We use Algorithm 2.2 on the test system with modified rates $\tilde{k}_{j}^{\text {hi }}$. This means $\left(N_{j}(t)\right)_{0 \leq t \leq t_{\text {sim }}}^{j=1, \ldots, N}$ are independent Poisson processes with parameters $\tilde{k}_{j}^{\text {hi }}$. To compute 
$R(t)$, we use (4-1) and sample $\chi_{j}(t)$ via (2-2). To compute $\tilde{R}(t)$ we use the unmodified Arrhenius rates $k_{j}^{\text {hi }}$ in (4-4). For each of $R(t), \tilde{R}(t)$ and $\hat{R}(t)$, the low-temperature rates $k_{j}=k_{j}^{\text {lo }}$ used in (4-1) and (4-5) are the same. We take $g_{j}=1$ for all $j$ and $\beta^{\text {hi }}=2.5, \beta^{\text {lo }}=10.0$. The variable $n$ was varied to compare the cases where the Eyring-Kramers rates $k_{j}^{\text {hi }}$ underestimate $\left(n=\frac{1}{2}\right)$, overestimate $\left(n=-\frac{1}{2}\right)$, and provide an exact estimate $(n=0)$ of the modified rates $\tilde{k}_{j}^{\text {hi }}$. Results are shown in Figures 1 and 2. The test system shows that $\tilde{R}(t)$ can overestimate $R(t)$ if the Eyring-Kramers rate deviates from the true rate at $\beta^{\text {hi }}$, while $\hat{R}(t)$ tends to provide a conservative estimate of $R(t)$.

\section{References}

[1] D. Aristoff, The parallel replica method for computing equilibrium averages of Markov chains, Monte Carlo Methods Appl. 21 (2015), no. 4, 255-273. MR 3433037 Zbl 1329.65005

[2] D. Aristoff and T. Lelièvre, Mathematical analysis of temperature accelerated dynamics, Multiscale Model. Simul. 12 (2014), no. 1, 290-317. MR 3176312 Zbl 1326.82018

[3] D. Aristoff, T. Lelièvre, and G. Simpson, The parallel replica method for simulating long trajectories of Markov chains, Appl. Math. Res. Express. (2014), no. 2, 332-352. MR 3266702 Zbl 1315.65010

[4] N. Berglund and S. Dutercq, The Eyring-Kramers law for Markovian jump processes with symmetries, J. Theor. Prob. (2015), 1-40.

[5] A. Binder, T. Lelièvre, and G. Simpson, A generalized parallel replica dynamics, J. Comput. Phys. 284 (2015), 595-616. MR 3303634

[6] S. T. Chill and G. Henkelman, Molecular dynamics saddle search adaptive kinetic monte carlo, J. Chem. Phys. 140 (2014), no. 21, 214110.

[7] W. E, W. Ren, and E. Vanden-Eijnden, Simplified and improved string method for computing the minimum energy paths in barrier-crossing events, J. Chem. Phys. 126 (2007), no. 16, 164103.

[8] P. Hanggi, P. Talkner, and M. Borkovec, Reaction-rate theory: fifty years after Kramers, Rev. Modern Phys. 62 (1990), no. 2, 251-341. MR 1056234

[9] G. Henkelman and H. Jónsson, Improved tangent estimate in the nudged elastic band method for finding minimum energy paths and saddle points, J. Chem. Phys. 113 (2000), no. 22, 9978-9985.

[10] G. Henkelman, B. P. Uberuaga, and H. Jónsson, A climbing image nudged elastic band method for finding saddle points and minimum energy paths, J. Chem. Phys. 113 (2000), no. 22, 9901-9904.

[11] C. Le Bris, T. Lelièvre, M. Luskin, and D. Perez, A mathematical formalization of the parallel replica dynamics, Monte Carlo Methods Appl. 18 (2012), no. 2, 119-146. MR 2926765 Zbl 1243.82045

[12] T. Lelièvre and F. Nier, Low temperature asymptotics for quasistationary distributions in a bounded domain, Anal. PDE 8 (2015), no. 3, 561-628. MR 3353826 Zbl 1320.58021

[13] R. A. Olsen, G. J. Kroes, G. Henkelman, A. Arnaldsson, and H. Jónsson, Comparison of methods for finding saddle points without knowledge of the final states, J. Chem. Phys. 121 (2004), no. 20, 9776-9792.

[14] D. Perez, B. P. Uberuaga, Y. Shim, J. G. Amar, and A. F. Voter, Accelerated molecular dynamics methods: introduction and recent developments, Ann. Rep. Comp. Chem. 5 (2009), 79-98. 
[15] G. Simpson and M. Luskin, Numerical analysis of parallel replica dynamics, ESAIM Math. Model. Numer. Anal. 47 (2013), no. 5, 1287-1314. MR 3100764 Zbl 1298.65016

[16] A. F. Voter, F. Montalenti, and T. C. Germann, Extending the time scale in atomistic simulation of materials, Ann. Rev. Materials Research 32 (2002), no. 1, 321-346.

[17] L. Xu and G. Henkelman, Adaptive kinetic monte carlo for first-principles accelerated dynamics, J. Chem. Phys. 129 (2008), no. 11, 114104.

[18] L. Xu, D. Mei, and G. Henkelman, Adaptive kinetic monte carlo simulation of methanol decomposition on cu(100), J. Chem. Phys. 131 (2009), no. 24, 244520.

Received June 30, 2015.

DAVID ARISTOFF: aristoff@rams.colostate.edu

Department of Mathematics, Colorado State University, 221 Weber Hall,

Fort Collins, CO 80523-1894, United States

SAMUEL T. CHILl: sam.chill@quantumwise.com

QuantumWise A/S, Austin, TX 78749, United States

GIDEON SIMPSON: simpson@math.drexel.edu

Department of Mathematics, Drexel University, Korman Center, 33rd and Market Streets,

Philadelphia, PA 19104, United States 


\title{
Communications in Applied Mathematics and Computational Science
}

\author{
msp.org/camcos
}

EDITORS

MANAGING EDITOR

John B. Bell

Lawrence Berkeley National Laboratory, USA

jbbell@lbl.gov

\section{BOARD OF EDITORS}

\begin{tabular}{|c|c|c|c|}
\hline Marsha Berger & $\begin{array}{l}\text { New York University } \\
\text { berger@cs.nyu.edu }\end{array}$ & Ahmed Ghoniem & $\begin{array}{l}\text { Massachusetts Inst. of Technology, USA } \\
\text { ghoniem@mit.edu }\end{array}$ \\
\hline Alexandre Chorin & $\begin{array}{l}\text { University of California, Berkeley, USA } \\
\text { chorin@math.berkeley.edu }\end{array}$ & Raz Kupferman & $\begin{array}{l}\text { The Hebrew University, Israel } \\
\text { raz@math.huji.ac.il }\end{array}$ \\
\hline Phil Colella & $\begin{array}{l}\text { Lawrence Berkeley Nat. Lab., USA } \\
\text { pcolella@lbl.gov }\end{array}$ & Randall J. LeVeque & $\begin{array}{l}\text { University of Washington, USA } \\
\text { rj1@amath.washington.edu }\end{array}$ \\
\hline Peter Constantin & $\begin{array}{l}\text { University of Chicago, USA } \\
\text { const@cs.uchicago.edu }\end{array}$ & Mitchell Luskin & $\begin{array}{l}\text { University of Minnesota, USA } \\
\text { luskin@umn.edu }\end{array}$ \\
\hline Maksymilian Dryja & $\begin{array}{l}\text { Warsaw University, Poland } \\
\text { maksymilian.dryja@acn.waw.pl }\end{array}$ & Yvon Maday & $\begin{array}{l}\text { Université Pierre et Marie Curie, France } \\
\text { maday@ann.jussieu.fr }\end{array}$ \\
\hline M. Gregory Forest & $\begin{array}{l}\text { University of North Carolina, USA } \\
\text { forest@amath.unc.edu }\end{array}$ & James Sethian & $\begin{array}{l}\text { University of California, Berkeley, USA } \\
\text { sethian@ math.berkeley.edu }\end{array}$ \\
\hline Leslie Greengard & $\begin{array}{l}\text { New York University, USA } \\
\text { greengard@cims.nyu.edu }\end{array}$ & Juan Luis Vázquez & $\begin{array}{l}\text { Universidad Autónoma de Madrid, Spain } \\
\text { juanluis.vazquez@uam.es }\end{array}$ \\
\hline Rupert Klein & $\begin{array}{l}\text { Freie Universität Berlin, Germany } \\
\text { rupert.klein@pik-potsdam.de }\end{array}$ & Alfio Quarteroni & $\begin{array}{l}\text { Ecole Polytech. Féd. Lausanne, Switzerland } \\
\text { alfio.quarteroni@epfl.ch }\end{array}$ \\
\hline \multirow[t]{2}{*}{ Nigel Goldenfeld } & $\begin{array}{l}\text { University of Illinois, USA } \\
\text { nigel@uiuc.edu }\end{array}$ & Eitan Tadmor & $\begin{array}{l}\text { University of Maryland, USA } \\
\text { etadmor@cscamm.umd.edu }\end{array}$ \\
\hline & & Denis Talay & $\begin{array}{l}\text { INRIA, France } \\
\text { denis.talay@inria.fr }\end{array}$ \\
\hline
\end{tabular}

\section{PRODUCTION}

production@msp.org

Silvio Levy, Scientific Editor

See inside back cover or msp.org/camcos for submission instructions.

The subscription price for 2016 is US $\$ 95 /$ year for the electronic version, and $\$ 135 /$ year $(+\$ 15$, if shipping outside the US) for print and electronic. Subscriptions, requests for back issues from the last three years and changes of subscribers address should be sent to MSP.

Communications in Applied Mathematics and Computational Science (ISSN 2157-5452 electronic, 1559-3940 printed) at Mathematical Sciences Publishers, 798 Evans Hall \#3840, c/o University of California, Berkeley, CA 94720-3840, is published continuously online. Periodical rate postage paid at Berkeley, CA 94704, and additional mailing offices.

CAMCoS peer review and production are managed by EditFLOW ${ }^{\circledR}$ from MSP.

\section{PUBLISHED BY}

mathematical sciences publishers

nonprofit scientific publishing

http://msp.org/

(C) 2016 Mathematical Sciences Publishers 


\section{Communications in Applied Mathematics and Computational Science}

Vol. 11

no. 2

2016

A real-space Green's function method for the numerical solution of

Maxwell's equations

Boris Lo, Victor Minden and Phillip Colella

Analysis of estimators for Adaptive Kinetic Monte Carlo

David Aristoff, Samuel T. Chill and Gideon Simpson

Comparison of continuous and discrete-time data-based modeling for hypoelliptic systems

Fei Lu, Kevin K. Lin and Alexandre J. Chorin

Hydrodynamics of suspensions of passive and active rigid particles: a rigid multiblob approach

Florencio Balboa Usabiaga, Bakytzhan Kallemov, Blaise

Delmotte, Amneet Pal Singh Bhalla, Boyce E. Griffith and AleKsandar Donev 